\title{
Working Memory Capacity and Visual-Verbal Cognitive Load Modulate Auditory-Sensory Gating in the Brainstem: Toward a Unified View of Attention
}

Patrik Sörqvist, Stefan Stenfelt and Jerker Rönnberg

\section{Linköping University Post Print}

N.B.: When citing this work, cite the original article.

Original Publication:

Patrik Sörqvist, Stefan Stenfelt and Jerker Rönnberg, Working Memory Capacity and VisualVerbal Cognitive Load Modulate Auditory-Sensory Gating in the Brainstem: Toward a Unified View of Attention, 2012, Journal of cognitive neuroscience, (24), 11, 2147-2154. http://dx.doi.org/10.1162/jocn_a_00275

Copyright: Massachusetts Institute of Technology Press (MIT Press): STM Titles http://mitpress.mit.edu/main/home/default.asp?sid=19E29805-C0A0-4642-8ECD-BACF5ADFF807

Postprint available at: Linköping University Electronic Press

http://urn.kb.se/resolve?urn=urn:nbn:se:liu:diva-85290 


\title{
Working Memory Capacity and Visual-Verbal Cognitive Load Modulate Auditory-Sensory Gating in the Brainstem: Toward a Unified View of Attention
}

\author{
Patrik Sörqvist $^{1,2,3}$, Stefan Stenfelt ${ }^{2,3}$, and Jerker Rönnberg ${ }^{2,3}$
}

\begin{abstract}
Two fundamental research questions have driven attention research in the past: One concerns whether selection of relevant information among competing, irrelevant, information takes place at an early or at a late processing stage; the other concerns whether the capacity of attention is limited by a central, domain-general pool of resources or by independent, modality-specific pools. In this article, we contribute to these debates by showing that the auditoryevoked brainstem response (an early stage of auditory processing)
\end{abstract}

\section{INTRODUCTION}

Two fundamental research questions have driven attention research during the past four decades. One concern is whether the filtering of irrelevant stimuli takes place at an early or at a late processing stage. Proponents of the early selection view argue that irrelevant stimuli are filtered (or attenuated) at a perceptual level (e.g., Treisman, 1964), whereas those who adhere to a late selection view argue that goal-irrelevant stimuli are perceptually processed in full but filtered at a postperceptual or response level (e.g., Deutsch \& Deutsch, 1963). The other question concerns how much information can be simultaneously attended to. Some theorists have argued in favor of a view according to which people have several modality-specific limited pools of resources that operate independently (see Wickens, 2008 , for a review), whereby depletion of visual resources does not compromise auditory processing and vice versa. In contrast, others have argued that people have a single, central, domain-general pool of resources at their disposal (e.g., Kahneman, 1973) and when they engage in a demanding task, this multipurpose pool of resources saturates, leaving less room for additional processing regardless of the task domain.

These debates have converged more recently into a unified view of attention whereby late/central processing interacts with early/sensory processing. One source of evidence comes from working memory load manipulations. High working memory load in a visual-verbal focal task reduces the likelihood that irrelevant sound will capture

${ }^{1}$ University of Gävle, ${ }^{2}$ Linnaeus Centre HEAD, Swedish Institute for Disability Research, ${ }^{3}$ Linköping University to task-irrelevant sound decreases as a function of central working memory load (manipulated with a visual-verbal version of the $n$-back task). Furthermore, individual differences in central/ domain-general working memory capacity modulated the magnitude of the auditory-evoked brainstem response, but only in the high working memory load condition. The results support a unified view of attention whereby the capacity of a late/central mechanism (working memory) modulates early precortical sensory processing.

attention (SanMiguel, Corral, \& Escera, 2008) and interfere with the task (e.g., Kim, Kim, \& Chun, 2005). Moreover, in a dual-task setting, high working memory load sometimes makes a separate, concurrent task more susceptible to distraction from irrelevant information (Lavie, Hirst, de Fockert, \& Viding, 2004) and sometimes less susceptible (Park, Kim, \& Chun, 2007), depending on the relation between the task material and distracting material. A second source of evidence comes from neuroscientific studies. Increasing auditory working memory load decreases activity in brain areas serving visual processing (Klemen, Büchel, Bühler, Menz, \& Rose, 2010) and vice versa (Zhang, Chen, Yuan, Zhang, \& He, 2006; see also Mozolic et al., 2008; Dyson, Alain, \& He, 2005). Moreover, prefrontal areas (usually associated with working memory) modulate cortical sensory processing areas in the service of selective attention (Gazzaley \& Nobre, 2012). A third source of evidence comes from individual difference studies. Individuals with high working memory capacity (WMC), as measured by complex-span tasks that tap into a domaingeneral construct (Kane et al., 2004), are more able to divide attention across multiple channels (Colflesh \& Conway, 2007) and more able to maintain focus in selective attention situations (Kane, Bleckley, Conway, \& Engle, 2001) in comparison with their low capacity counterpart. Furthermore, high-WMC individuals are less susceptible to auditory distraction in visual-verbal task settings (Sörqvist, Nöstl, \& Halin, 2012) as well as in auditory-verbal task settings (Sörqvist \& Rönnberg, 2012; Stenfelt \& Rönnberg, 2009; Rönnberg, Rudner, Foo, \& Lunner, 2008; Conway, Cowan, \& Bunting, 2001). Findings such as these suggest that individuals with a large pool of central cognitive 
resources (i.e., high WMC) have superior attention abilities and have led some theorists to argue that WMC is equivalent to the capacity of attention (Cowan, 2005). Taken together, these studies support a unified approach to attention and capacity whereby the capacity of a central mechanism (working memory) modulates sensory gating, both within the target modality and across modalities. In this article, we set out to test whether central working memory modulates auditory-perceptual filtering of irrelevant sound and, in particular, whether this filtering takes place at an early (sensory) processing stage.

When sound reaches the ear and the cochlea, it is transformed into a neural signal that is transmitted through the brainstem via, for example, the olivary complex, before it reaches the thalamus and ultimately the auditory cortex. This neural activity can be measured by evoked potentials known as auditory brainstem response (ABR). The ABR is usually measured by surface electrodes with one electrode at the vertex or in the middle of the forehead, one behind the ear on the mastoid or on the earlobe, and one ground electrode on the opposite of the head. Normally, the ABR is elicited by a short transient sound (click stimulation) but short tone-burst stimuli can also be used. The response waveform peaks are labeled I to VII, where Wave V usually has the greatest amplitude and therefore is the most robust. Wave $\mathrm{V}$ appears usually between 6 and $8 \mathrm{msec}$ after stimulus onset and is the one mostly used for audiological purposes (Pratt, 2003).

Physiological evidence indicates that selective attention (e.g., focusing on one tone while ignoring another tone) modulates the activity of the outer hair cells within the cochlea to facilitate processing of the target stimuli (de Boer \& Thornton, 2007; Giard, Collet, Bouchet, \& Pernier, 1994; Meric \& Collet, 1992), although the generality of this phenomenon has been questioned (Michie, LePage, Solowij, Haller, \& Terry, 1996). Similar attention-related changes of the ABR have also been shown (Althen, Grimm, \& Escera, 2011; Hoormann, Falkenstein, \& Hohnsbein, 1994; Galbraith \& Arroyo, 1993; Bauer \& Bayles, 1990), although some attempts have failed (Hirschhorn \& Michie, 1990) and the effect appears to have a limited generality (Hoormann, Falkenstein, \& Hohnsbein, 2000). In all, there is indication of attention-related top-down modulation (or suppression) of the auditory neural signal at precortical processing stages, although the results have been inconsistent. One potential source of this inconsistency is the demands of the tasks employed. Previous studies have, to the best of our knowledge, only compared ABRs for a condition in which the sound is attended with another condition in which attention is directed elsewhere (either to visual targets or to another sound) and the sound is ignored. The demands of the focal (to-be-attended) task have never been systematically manipulated to test whether this manipulation modulates the responses to the to-be-ignored sound. One possibility is that, with higher demands of the focal task, attention resources are withdrawn from extra task processing, decreasing responsiveness to task-irrelevant sound.
The purpose of the present experiment was to test whether working memory modulates auditory-sensory gating of irrelevant sound in the brainstem. To this end, we compared ABR magnitudes for a task-irrelevant sound during three levels of central working memory load (as manipulated with a visual-verbal version of the $n$-back task in which participants maintain and continuously update a set of $n$ items in working memory; Braver et al., 1997). For comparison purposes, we also added a condition in which the participants were requested to actively listen to the sound. We expected the ABR magnitude to be highest in the active listening condition and to decrease as a function of working memory load. Moreover, the relationship between WMC and ABR magnitude was also investigated. On the basis of previous findings, one may expect greater suppression of irrelevant stimuli for high-WMC individuals, as they presumably are more able to engage attention resources in the focal task and especially so in the high-load condition.

\section{METHODS}

\section{Participants}

A total of 33 students at Linköping University took part in this study in exchange for a small honorarium. All had normal hearing (screened) and reported normal or correctedto-normal vision and Swedish as their native language.

\section{Materials and Apparatus}

n-back

Three conditions of this task were created. In each condition, the participants were visually presented with a sequence of letters pseudorandomly drawn from a closed set of seven letters (w s r k q t m). Each letter was presented 10 times individually with a 3000-msec duration in the center of a computer screen. The ISI (offset to onset) was $500 \mathrm{msec}$. The participants were told to strike the space bar on the computer keyboard when the presented letter was the same as the letter presented $n$ letters back in the sequence, and they were told to withhold responses when this was not the case. The presented letter matched the letter $n$ letters back a total of 14 times (20\% of the letters) in each version of the task. The size of $n$ was 1,2 , or 3 ; one size for each task condition.

\section{Complex-span Tasks}

Three tasks were used to asses WMC. In the operation span task (Turner \& Engle, 1989) and in the size-comparison span task (Sörqvist, Ljungberg, \& Ljung, 2010), the participants viewed lists of visually presented to-be-serially recalled words, and between each word they undertook a distractor activity. In operation span task, the distractor activity was to solve a mathematical expression (e.g., "Is $2 \times 4 / 3=2$ ?'), and, in size comparison span task, to make a size comparison (e.g., "Is ELEPHANT larger than MOUSE?'). Answers were given by pressing either of two buttons on the 
keyboard (one for "yes" and one for "no"). In both tasks, the to-be-recalled words were presented for $800 \mathrm{msec}$. The ISI (offset to onset) between words and operations/ comparisons was $500 \mathrm{msec}$. The number of words to-berecalled varied between 2 and 6 . There were two lists of each list length, and the length of the lists increased throughout the tasks. At the end of each list, the computer probed the participants to recall the words in order of presentation by typing on the computer keyboard. The tasks were scored using a strict serial recall criterion (i.e., points were assigned to each word that was reported in the correct serial position only), and the scores for each list were multiplied with the total number of words in that list to balance list difficulty (as in Sörqvist et al., 2012). For reading span (Daneman \& Carpenter, 1980), the participants viewed lists of three-word sentences (the words were presented consecutively), and their task was to decide whether or not the sentence was absurd or normal (e.g., "fish smoked cigar") by pressing either a button for "absurd" or a button for "normal" on the keyboard. At the end of each list, the participants were probed to recall the first or the last word of each sentence. Each word was presented for 800 msec with an ISI (offset to onset) between words of $500 \mathrm{msec}$. The number of sentences varied between 2 and 6 and increased throughout the task. There were two lists of each list length. Recall was done the same way as in the other two WMC tasks. The task was scored using a free recall criterion (i.e., points were assigned to each accurately reported word regardless of position), and the scores for each list were multiplied by the total number of words in that list to balance list difficulty. Finally, all three tasks were then used to form an index of WMC by calculating an average score across the three tasks for each participant individually.

\section{Sound}

Two tone bursts, presented binaurally, were used for stimulation, a $1.0-\mathrm{kHz}$ stimulus (standard tone) and a $1.2-\mathrm{kHz}$ stimulus (deviant tone). The stimulus consisted of four cycles of the tone: one cycle rise, two cycles plateau, and one cycle fall. The tone burst length was $4.0 \mathrm{msec}$ for the $1.0-\mathrm{kHz}$ stimulus and $3.33 \mathrm{msec}$ for the $1.2 \mathrm{-kHz}$ stimulus. The repetition rate for the stimulation was $39.9 \mathrm{stim} / \mathrm{sec}$ and the stimulus block consisted of forty $1.0-\mathrm{kHz}$ stimuli or forty 1.2-kHz stimuli (block time of $1002.5 \mathrm{msec}$ ). The probability of a deviant sound was 0.1 , and the total number of blocks was 240 with 24 deviant blocks and 216 standard blocks, resulting in a total test stimulus of $240.6 \mathrm{sec}$. Two deviant blocks never occurred without at least one normal block in-between. The stimulation level was set at $60 \mathrm{~dB}$ SPL.

\section{Brainstem Response Audiometry}

The evoked potentials from the brainstem were measured by disposable surface electrodes positioned at the forehead and mastoid with a ground electrode on the temple. The electrodes were connected to a preamplifier and sub- sequently to an $\mathrm{A} / \mathrm{D}$ converter (National Instruments USB 6212, Austin, TX) coupled to a computer. Although the sound was presented diotically (same signal to both ears), the evoked potentials were obtained from the left ear only. The incoming signal was sampled at $32 \mathrm{kHz}$ and stored on the computer hard drive. An alternating polarity procedure was used whereby every second tone burst is presented with positive phase (condensation) and every other second tone burst with negative phase (rarefaction); the repetition rate of the tone bursts was, as stated above, set at 39.9 stim/sec. All measurements with excessive noise (traces containing signals above $40 \mu \mathrm{V}$ ) were removed before the analysis. For each condition, each participant was tested with 4320 condensation $1.0-\mathrm{kHz}$ tone burst stimuli and 4320 rarefaction $1.0 \mathrm{kHz}$-tone burst stimuli. These tone bursts were individually averaged as condensation and rarefaction responses and compared for conformity; they were subsequently averaged to one trace for analysis. As electrical stimulus artifacts affected the ABR response, data points before $4 \mathrm{msec}$ after stimulus onset could not be analyzed, and there were no attempt to identify other peaks beside Wave V. Here, the amplitude of Wave $\mathrm{V}$ was estimated as the peak of Wave $\mathrm{V}$ (occurring typically between 6 and $7 \mathrm{msec}$ after stimulus onset; see Figure 1B) compared with the dip occurring between 9.5 and $10.5 \mathrm{msec}$ in the ABR trace. Because there are interindividual differences in the Wave V estimate, the amplitude was normalized to the mean amplitude for the four test conditions; this facilitates comparison between participants of the relative amplitudes for the four conditions.

\section{Design and Procedure}

The experiment consisted of two sessions. The participants first undertook the three WMC tasks, the order of which was counterbalanced between participants. They sat alone in a quiet room in front of a laptop computer that handled stimulus presentation and recording of responses. They then came back for a second session for four test conditions. First, they did the three conditions of $n$-back (the order of the conditions was counterbalanced between participants) performed on a laptop computer. Paralleling the $n$-back test, a 240.6-sec ABR test was conducted as described above. After the last condition of $n$-back, the experiment ended with an active listening condition. During this condition, the same sound stimulation (but no $n$-back task) was provided and the ABR was recorded while the participants were requested to count the number of deviating tone blocks, to press the space bar after each occurrence, and to report the total count at the end of the condition.

\section{RESULTS}

\section{Behavioral Data}

WMC and n-back

The mean WMC score, expressed as a probability value, was $.59(S D=0.14$, range $.31-.81)$. $n$-Back data from two 

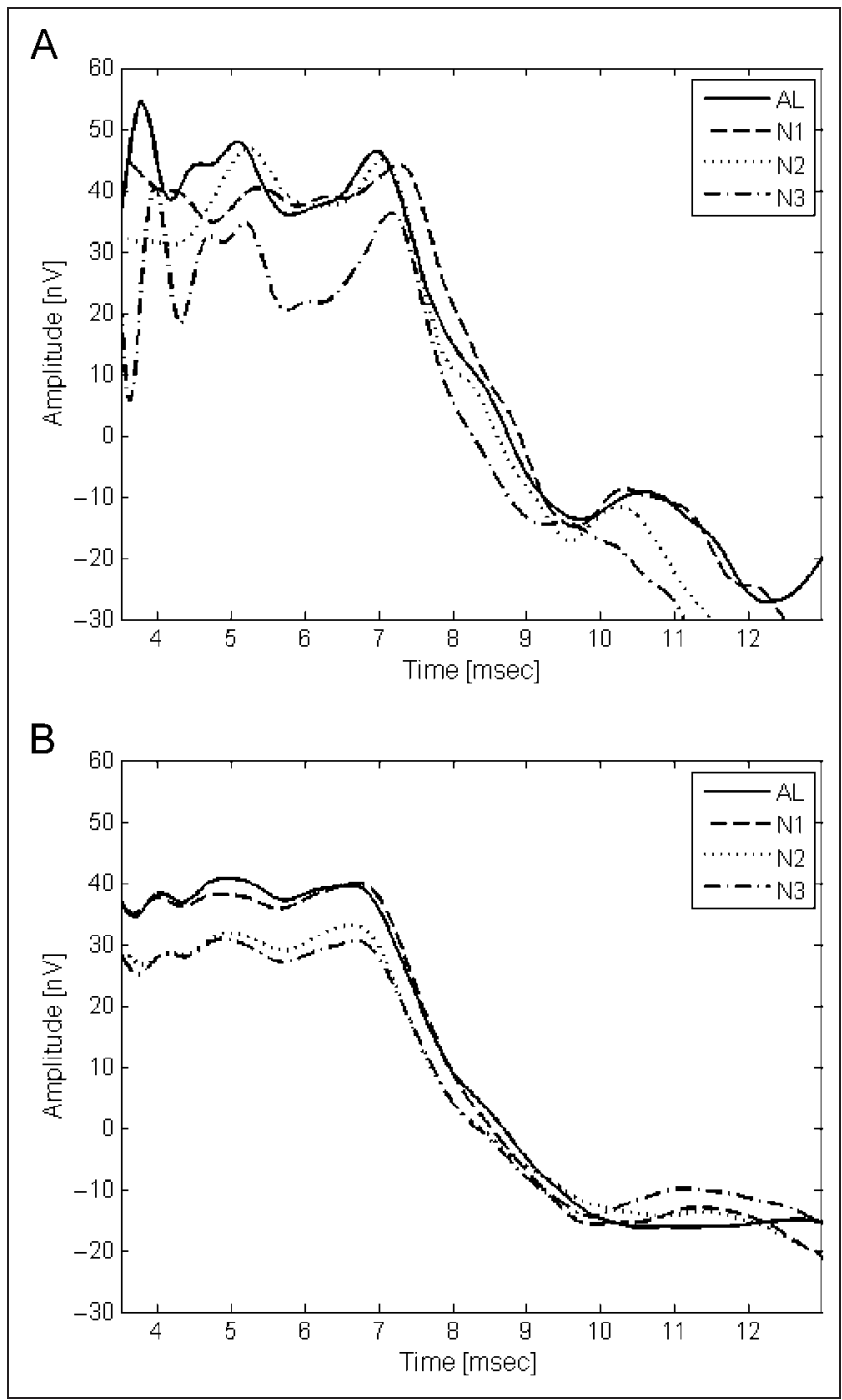

Figure 1. The figure shows the ABRs as a function of time for one representative participant (A) and for the four conditions averaged over all participants (B). The Wave V is visible at around $6.8 \mathrm{msec}$ in all traces, and its magnitude was calculated as the difference between the peak of Wave $\mathrm{V}$ and the dip at around $10 \mathrm{msec}$. The four conditions were active listening (AL, solid line), the 1-back task (N1, dashed line), 2-back task (N2, dotted line), and the 3-back task (N3, dash-dotted line).

participants were lost because of technical failure. Mean performance $(n=31)$, expressed as a probability score, was $.99(S D=.01), .99(S D=.02)$, and $.89(S D=.06)$ on the 1-back, 2-back, and 3-back version of the $n$-back task, respectively. Alpha was set to .05 in all statistical analyses. A one-way repeated-measures analysis of variance confirmed a significant difference between the high load condition (3-back) and the other two conditions, $F(2,58)=$ 102.81, MSE $=0.001, p<.001, \eta_{\mathrm{p}}^{2}=.77$. Follow-up $t$ tests revealed a significant difference between 3 -back and 2-back, $t(30)=11.04, p<.001$ and between 3-back and 1-back, $t(30)=9.98, p<.001$, but not between 1-back and 2-back, $t(30)=0.78, p=.440$.
Counting Deviating Tones during Active Listening

The sound consisted of a sequence of tone blocks (1002.50 msec in duration). A standard tone $(1.0 \mathrm{kHz})$ was presented repeatedly (40 times) in 216 blocks, and a deviating tone $(1.2 \mathrm{kHz})$ was presented repeatedly (40 times) in 24 blocks. In the active listening condition, the participants were requested to count the deviating tone blocks. The participants performed near ceiling on this task. Twenty-nine of 33 participants responded accurately (i.e., 24), and the other four reported 25, 25, 23, and 22 deviating tone blocks, respectively.

\section{Auditory-Evoked Brainstem Response}

Data from two participants were lost because of technical failure (not the same as above). The ABR from a representative participant in the four experimental conditions (active listening, low, intermediate, and high visualverbal cognitive load) as a function of time is reported in Figure 1A and the grand averaged ABR for the four experimental conditions is reported in Figure 1B. The Wave $\mathrm{V}$ visible at around $6.8 \mathrm{msec}$ for all traces in Figure 1 is the most prominent and consistent component of the $\mathrm{ABR}$, and its magnitude is therefore used in the current analysis. Also apparent in Figure 1 are the differences in ABR magnitudes at latencies shorter than Wave V (between 3.5 and $6.8 \mathrm{msec}$ ). However, as the peaks before Wave $\mathrm{V}$ were not identifiable in the individual traces, this part of the ABR trace is not analyzed in the current study.

In what follows, $\mathrm{ABR}$ magnitude refers to the magnitude of Wave V of the ABR trace. As can be seen in Figure 2, the normalized magnitude of Wave $\mathrm{V}$ (in response to the $1.0 \mathrm{kHz}$ standard tone) was highest in the active listening condition and declined as a function of increasing cognitive

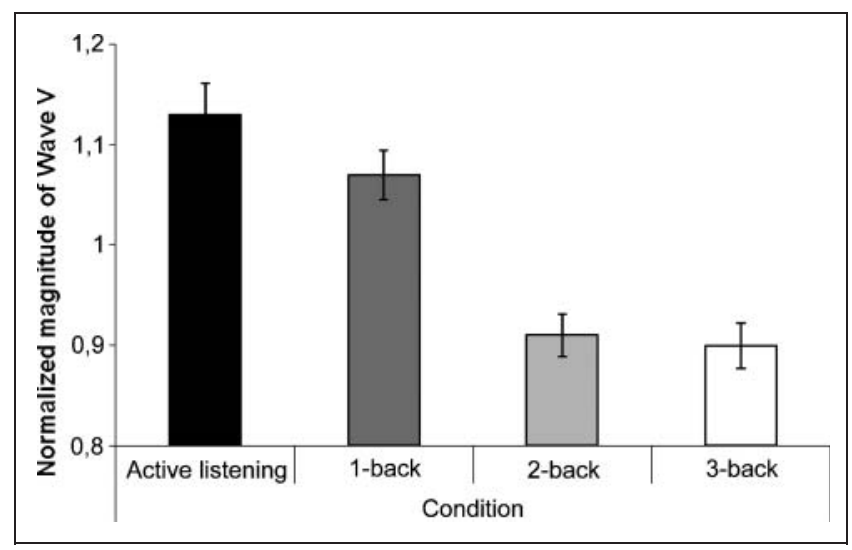

Figure 2. The figure shows the ABR (normalized Wave V magnitude) to sound when the participants were asked to actively listen to the sound only, and when the sound was played in the background while the participants performed three versions of the $n$-back task with varying cognitive load (1-back $=$ low load, 2-back $=$ intermediate load, 3-back $=$ high load). Error bars show the SEMs. 
Table 1. The Correlation between ABRs (Wave V) under Four Experimental Conditions, WMC, and Scores of Three Versions of $n$-back with Various Cognitive Load

\begin{tabular}{|c|c|c|c|c|c|c|c|}
\hline Measure & 1. & 2. & 3. & 4. & 5. & 6. & 7. \\
\hline 1. $\mathrm{ABR}$ in active listening condition & 1 & & & & & & \\
\hline 2. $\mathrm{ABR}$ in 1-back condition & -.23 & 1 & & & & & \\
\hline 3. ABR in 2-back condition & $-.47 * *$ & $-.39 *$ & 1 & & & & \\
\hline 4. $\mathrm{ABR}$ in 3-back condition & $-.62 * *$ & -.31 & .11 & 1 & & & \\
\hline 5. WMC index & .17 & .12 & .12 & $-.47 * *$ & 1 & & \\
\hline 6. 1-Back score & -.05 & -.26 & .19 & .15 & -.08 & 1 & \\
\hline 7. 2-Back score & .04 & -.10 & .17 & -.11 & .25 & -.21 & 1 \\
\hline 8. 3-Back score & -.20 & -.04 & .23 & .13 & .13 & -.06 & $.49 * *$ \\
\hline
\end{tabular}

load. This conclusion was supported by a repeated-measures $\operatorname{ANOVA}(n=31), F(3,90)=14.92, M S E=0.03, p<.001$ $\eta_{\mathrm{p}}{ }^{2}=.33$. Bonferroni-adjusted follow-up $t$ tests revealed no significant difference between the active listening and 1-back condition, and no difference between the 2-back and 3-back condition. All other comparisons were significant at the $p<.001$ level.

The intercorrelations for the normalized magnitudes of Wave $\mathrm{V}$ in the four experimental conditions and their relations to WMC are reported in Table 1. Two findings are particularly noteworthy. First, higher WMC was associated with a lower ABR magnitude, but only in the high load condition, as hypothesized. Second, the ABR magnitude in active listening and in the low load condition was negatively related to the ABR magnitude in the conditions of higher visual-verbal cognitive load. This generally indicates that, as the cognitive demands changed, some participants modulated the ABR magnitude to a greater extent than others (those with greater magnitude in the 1-back condition tended to have lower magnitude in the 3-back condition). We used a residual analysis technique in the context of hierarchical regression analysis to analyze whether WMC could account for some of this variance. Specifically, we tested whether WMC is related to the change in ABR magnitude between the 1-back and the 3-back condition, because they represent the largest difference between two cognitive load conditions whereby the sound is taskirrelevant and should reveal how visual-verbal cognitive load modulates perceptual filtering of irrelevant sound. ABR magnitudes in the 3-back condition were selected as the dependent variable, ABR magnitudes in the 1-back condition were selected as the independent variable of the first step of the hierarchical regression analysis, and WMC scores were selected as independent variable in the second step of the analysis. The residual variance left to be explained in the second step of this analysis represents the difference between ABR magnitude in the 1-back condition and ABR magnitude in the 3-back condition. Hence, if
WMC is related to this residual variance, the results would indicate that WMC is related to the modulation of the ABR across conditions. Indeed, WMC explained a significant and unique part of the variance when added in the second step of the analysis, $\Delta R^{2}=.19, \beta=-.44, t(28)=-2.72, p=$ .011. As correlations are sensitive to outliers and may well occur as a result of a single value that deviates from the rest of the sample, we conducted a control analysis with outliers removed. The correlation between the residual variance and WMC was even stronger when outliers $(z>$ 2.00) were excluded, $r(26)=-.52, p<.01$, and further reinforce the conclusion that higher WMC is related to a greater modulation of the ABR magnitude (Figure 3).

\section{DISCUSSION}

The experimental manipulation of this study suggests a causal link between working memory and early sensory gating: The magnitude of auditory-evoked brainstem responses (ABRs) to irrelevant sound decreases as a function of increasing visual-verbal working memory load. Also, individual differences in WMC were negatively related to the magnitude of the ABR. These results are particularly supportive of a unitary view of attention whereby a late/central mechanism (working memory) suppresses-to the extent of its capacity-irrelevant sensory information (Klemen et al., 2010; Mozolic et al., 2008; SanMiguel et al., 2008; Zhang et al., 2006; Dyson et al., 2005). Attention seems to be used as a "gatekeeper" in the service of working memory to protect its capacity-limited processing space from overload (Awh, Vogel, \& Oh, 2006) — as is particularly needed when working memory task-load is high-and, at least in this study, operates at an early processing stage. This framework offers an explanation as to why individuals with high WMC are less susceptible to auditory distraction (e.g., Sörqvist et al., 2012; Conway et al., 2001): Higher WMC enables greater control of incoming irrelevant auditory 
Figure 3. The figure shows $z$ values (outliers removed) for the relationship between WMC and the change in ABRs (Wave V) between the low load condition (1-back) and the high load condition (3-back). The change scores ( $y$ axis) are the residual variance left to be explained when ABRs in the low load condition was partialed out from ABRs in the high load condition. Note that the smaller values on the $y$ axis represent a larger change in the negative direction (a larger decrease of the ABR).

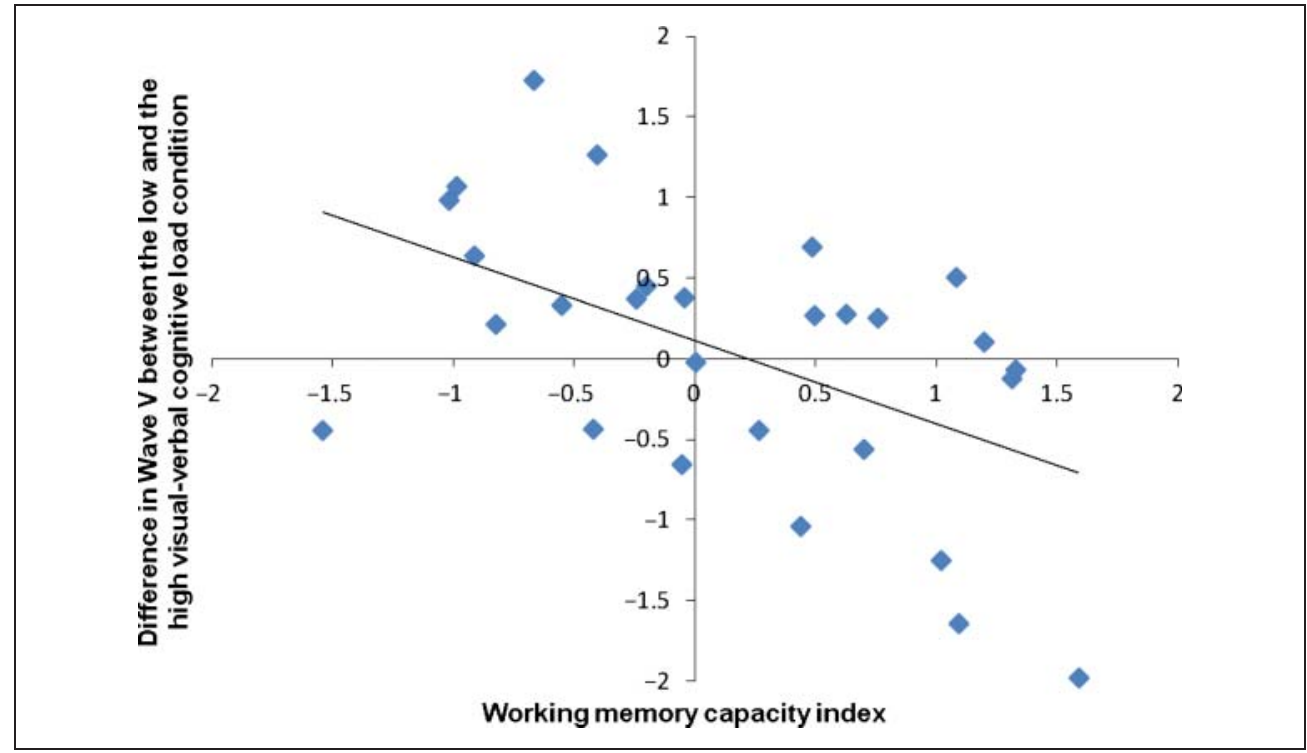

stimulation by suppression of early auditory-sensory processing.

Interestingly, the experiment reported here shows that high working memory load decreases responsiveness to irrelevant information, whereas other studies have demonstrated the opposite direction of this relationship whereby high working memory load increases susceptibility to distraction (e.g., Dalton, Santangelo, \& Spence, 2009; Lavie et al., 2004). A key difference between these latter studies (e.g., Lavie et al., 2004) and the one reported here is that they manipulated working memory load in a dualtask situation (e.g., maintaining items in working memory while performing an unrelated visual search task), and in this dual-task situation, the concurrent nonworking memory task (i.e., visual search) becomes more susceptible to distraction under high working memory load, as responsiveness to irrelevant information increases. Here, we manipulated working memory load in a single-task situation, and in this condition, processing of irrelevant information decreases under high working memory load. It appears as if working memory constrains attention to the focal task and protects it from distraction, but when there are two separate focal tasks and when one of those demands working memory resources, the other task is left unprotected. Taken together, both lines of research converge on the view that working memory plays an important role in top-down control of selective attention (cf. Klemen et al., 2010).

We propose a speculative neuroscientific explanation for the relationship between working memory and attention observed here. Because WMC is strongly associated with prefrontal lobe functioning (Kane \& Engle, 2002), one possibility is that the prefrontal lobe (in particular, the dorsolateral pFC, BA 9, BA 10, and BA 46; Collette et al., 1999) is the basis of a central mechanism that orchestrates an attention network that includes the brainstem and possibly suppresses incoming sound stimuli by sending inhibitory signals to the brainstem, in much the same way as the prefrontal lobe is involved in modulation of other cortex areas in the service of selective attention (Gazzaley \& Nobre, 2012). A possible mediating mechanism - between the pFC and the brainstem - is the cortical cholinergic system, which is influenced by prefrontal efferent projections and acts, top-down, to suppress processing of irrelevant stimuli at both cortical and subcortical levels (Sarter, Hasselmo, Bruno, \& Givens, 2005).

One possible reason why an effect of selective attention on ABR magnitude for unattended stimuli was found here, whereas it has been difficult to find this relationship in previous studies (e.g., Hoormann et al., 2000; Hirschhorn \& Michie, 1990), is that we manipulated the cognitive load of the focal activity. The present experiment suggests that the focal activity has to be quite demanding for the effect to take place, it is not enough just to attend to another modality (as proposed by the absence of a difference in ABR magnitude between the active listening condition and the 1-back condition, Figure 2). Because of this, one possible source of inconsistencies among previous findings is the difference in cognitive demand of the focal activity. It should be noted, though, that the relationship between working memory load and ABR magnitude is not linear. Rather, the effect of additional working memory load on ABR appears to attenuate rapidly after a certain level, as evidenced by the absence of a difference in ABR magnitude between the 2-back and the 3-back condition. As there was no difference between these conditions, it is unclear why WMC was only related to the ABR magnitude in the 3-back condition, not in the 2-back condition. One possibility, as suggested by the positive-yet nonsignificant - correlation between WMC and ABR magnitude in the 2-back condition, is that individuals with high WMC performed the 2-back task quite easily (while their low WMC counterpart also managed to perform the task well but with greater effort) and had additional resources available for processing the 
sound. In the 3-back condition, however, also those with higher capacity needed to constrain attention to the focal activity, thus resulting in a significant negative relationship between WMC and ABR magnitude, as those with higher WMC are more capable to focus attention than others. This change in direction of the relationship is also a possible explanation of the absence of a difference in ABR magnitude between the 2-back and the 3-back condition. Although it remains unclear why WMC was only related to ABR magnitude in the 3-back condition, the most important finding reported here is the relationship between WMC and the size of the difference in ABR magnitude between the low-load condition (1-back) and the high-load condition (3-back), consistent with the idea that the capacity of a central working memory mechanism constrains early sensory gating.

The normalized ABR magnitude in Figure 2 goes from 1.13 while attending the sound to 0.90 when attention is engaged in the high visual-verbal cognitive load task. This average change in brainstem response magnitude between active listening and high cognitive load is just below $2 \mathrm{~dB}$. Compared with the approximately $120 \mathrm{~dB}$ dynamic range of the human auditory system, this less than $2 \mathrm{~dB}$ decrease may be considered too low to be meaningful. However, the brainstem amplitude change is not equal to the change of the basilar membrane vibration amplitude; because of the compression in the neural system, it can correspond to several greater magnitude differences in the cochlea. The exact relation depends on the stimulus itself and sound pressure level. It also is not clear whether the effect seen is caused by a change in a mechanical response of the basilar membrane because of the action of the outer hair cells or caused by an up-/down-regulation of the neural system in the brainstem itself.

One way to differentiate between the two possibilities is to investigate the latency of Wave V of the ABR for the four conditions measured. If the outer hair cells are affected, greater amplitude should be followed by a shorter latency of Wave V. The latency of Wave V is calculated as the time between the onset of the tone burst and the peak of Wave $\mathrm{V}$. The average latency with our stimulus paradigm was between $6.75 \pm 0.40 \mathrm{msec}$ for active listening and $6.84 \pm 0.44 \mathrm{msec}$ for the 3 -back task. Even if this indicates an increase in latency with task difficulty, it was not significant and the 1-back latency was similar to the 3-back latency whereas the 2-back latency was similar to the active listening latency. Consequently, the modulation of the brainstem response amplitude seems to be caused by an up-/down-regulation of the neural brainstem system rather than an effect in the cochlea. Here, it is interesting to note that Hoormann et al. (2000) found an effect of attention on the ABR when attention was directed to the sound, whereas this study found an effect of attention (or cognitive load) on the ABR when attention was directed away from the sound. The two findings may well represent two different mechanisms. Possibly, attention can modulate the brainstem system in two ways: by inhibiting processing of ignored sound when visual-verbal load increases and by facilitating processing of attended sound. The slight (nonsignificant) increase in ABR magnitude in the active listening condition, relative to the 1-back condition, points in this direction (Figure 2).

In conclusion, the results reported in this article indicate a top-down modulation of auditory-sensory gating that takes place even before the stimuli reach a cortical level of the brain, and they are particularly supportive of a unified view of attention whereby a late/central mechanism (and its capacity) modulates early/sensory processing. The experiment reported here demonstrates a potentially very important causal relationship between cognitive and perceptual processes.

\section{Acknowledgments}

The research reported here was financially supported by a grant from Stiftelsen Riksbankens Jubileumsfond (P11-0617:1) awarded to Patrik Sörqvist. We are grateful to Mats Leandersson, Helena Torlofson, and Wycliffe Yumba at Linköping University for assistance in data collection.

Reprint requests should be sent to Patrik Sörqvist, Department of Building, Energy and Environmental Engineering, University of Gävle, SE-801 76 Gävle, Sweden, or via e-mail: patrik.sorqvist@ hig.se.

\section{REFERENCES}

Althen, H., Grimm, S., \& Escera, C. (2011). Fast detection of unexpected sound intensity decrements as revealed by human evoked potentials. PLOS ONE, 6, e28522.

Awh, E., Vogel, E. K., \& Oh, S.-H. (2006). Interactions between attention and working memory. Neuroscience, 139, 201-208.

Bauer, L. O., \& Bayles, R. L. (1990). Precortical filtering and selective attention: An evoked potential analysis. Biological Psychology, 30, 21-33.

Braver, T. S., Cohen, J. D., Nystrom, L. E., Jonides, J., Smith, E. E., \& Noll, D. C. (1997). A parametric study of prefrontal cortex involvement in human working memory. Neuroimage, 5 , 49-62.

Colflesh, G. J. H., \& Conway, A. R. A. (2007). Individual differences in working memory capacity and divided attention in dichotic listening. Psychonomic Bulletin E Review, 14, 699-703.

Collette, F., Salmon, E., Van der Linden, M., Chicherio, C., Belleville, S., Degueldre, C., et al. (1999). Regional brain activity during tasks devoted to the central executive of working memory. Cognitive Brain Research, 7, 411-417.

Conway, A. R. A., Cowan, N., \& Bunting, M. F. (2001). The cocktail party phenomenon revisited: The importance of working memory capacity. Psychonomic Bulletin \& Review, 8, 331-335.

Cowan, N. (2005). Working memory capacity. New York: Psychology Press.

Dalton, P., Santangelo, V., \& Spence, C. (2009). The role of working memory in auditory selective attention. Quarterly Journal of Experimental Psychology, 62, 2126-2132.

Daneman, M., \& Carpenter, P. (1980). Individual differences in working memory and reading. Journal of Verbal Learning E Verbal Behavior, 19, 450-466. 
de Boer, J., \& Thornton, A. R. D. (2007). Effect of subject task on contralateral suppression of click evoked otoacoustic emissions. Hearing Research, 233, 117-123.

Deutsch, J. A., \& Deutsch, D. (1963). Attention: Some theoretical considerations. Psychological Review, 70, 80-90.

Dyson, B. J., Alain, C., \& He, Y. (2005). Effects of visual attentional load on low-level auditory scene analysis. Cognitive, Affective, \& Behavioral Neuroscience, 5, 319-338.

Galbraith, G. C., \& Arroyo, C. (1993). Selective attention and brainstem frequency-following responses. Biological Psychology, 37, 3-22.

Gazzaley, A., \& Nobre, A. C. (2012). Top-down modulation: Bridging selective attention and working memory. Trends in Cognitive Sciences, 16, 129-135.

Giard, M. H., Collet, L., Bouchet, P., \& Pernier, J. (1994) Auditory selective attention in the human cochlea. Brain Research, 633, 353-356.

Hirschhorn, T. N., \& Michie, P. T. (1990). Brainstem auditory evoked potentials (BAEPs) and selective attention revisited Psychophysiology, 27, 495-512.

Hoormann, J., Falkenstein, M., \& Hohnsbein, J. (1994). Effect of selective attention on the latency of human frequencyfollowing potentials. NeuroReport, 15, 1609-1612.

Hoormann, J., Falkenstein, M., \& Hohnsbein, J. (2000). Early attention effects in human auditory-evoked potentials. Psychophysiology, 37, 29-42.

Kahneman, D. (1973). Attention and effort. Englewood Cliffs, NJ: Prentice-Hall.

Kane, M. J., Bleckley, M. K., Conway, A. R. A., \& Engle, R. W. (2001). A controlled-attention view of working-memory capacity. Journal of Experimental Psychology: General, 130, 169-183.

Kane, M. J., \& Engle, R. W. (2002). The role of prefrontal cortex in working-memory capacity, executive attention, and general fluid intelligence: An individual-differences perspective. Psychonomic Bulletin \& Review, 9, 637-671.

Kane, M. J., Hambrick, D. Z., Tuholski, S. W., Wilhelm, O., Payne, T. W., \& Engle, R. W. (2004). The generality of working memory capacity: A latent-variable approach to verbal and visuospatial memory span and reasoning. Journal of Experimental Psychology: General, 133, 189-217.

Kim, S.-Y., Kim, M.-S., \& Chun, M. M. (2005). Concurrent working memory load can reduce distraction. Proceedings of the National Academy of Sciences, 102, 16524-16529.

Klemen, J., Büchel, C., Bühler, M., Menz, M. M., \& Rose, M. (2010). Auditory working memory load impairs visual ventral stream processing: Toward a unified model of attentional load. Journal of Cognitive Neuroscience, 22, 437-446.

Lavie, N., Hirst, A., de Fockert, J. W., \& Viding, E. (2004). Load theory of selective attention and cognitive control. Journal of Experimental Psychology: General, 133, 339-354.

Meric, C., \& Collet, L. (1992). Visual attention and evoked otoacoustic emissions: A slight but real effect. International Journal of Psychophysiology, 12, 233-235.
Michie, P. T., LePage, E. L., Solowij, N., Haller, M., \& Terry, L. (1996). Evoked otoacoustic emissions and auditory selective attention. Hearing Research, 98, 54-67.

Mozolic, J. L., Joyner, D., Hugenschmidt, C. E., Peiffer, A. M., Kraft, R. A., Maldjian, J. A., et al. (2008). Cross-modal deactivations during modality-specific selective attention. BMC Neurology, 8, 35.

Park, S., Kim, M.-S., \& Chun, M. M. (2007). Concurrent working memory load can facilitate selective attention: Evidence for specialized load. Journal of Experimental Psychology: Human Perception and Performance, 33, 1062-1075.

Pratt, H. (2003). Human auditory electrophysiology. In L. Luxon, J. M. Martin, \& D. G. Stephens (Eds.), A textbook of audiological medicine: Clinical aspects of hearing and balance (pp. 271-288). London: Taylor and Francis.

Rönnberg, J., Rudner, M., Foo, C., \& Lunner, T. (2008). Cognition counts: A working memory system for ease of language understanding (ELU). International Journal of Audiology, 47, S171-S177.

SanMiguel, I., Corral, M.-J., \& Escera, C. (2008). When loading working memory reduces distraction: Behavioral and electrophysiological evidence from an auditory-visual distraction paradigm. Journal of Cognitive Neuroscience, 20, 1131-1145.

Sarter, M., Hasselmo, M. E., Bruno, J. P., \& Givens, B. (2005). Unraveling the attentional functions of cortical cholinergic inputs: Interactions between signal-driven and cognitive modulation of signal detection. Brain Research Review, 48, 98-111.

Sörqvist, P., Ljungberg, J. K., \& Ljung, R. (2010). A sub-process view of working memory capacity: Evidence from effects of speech on prose memory. Memory, 18, 310-326.

Sörqvist, P., Nöstl, A., \& Halin, N. (2012). Working memory capacity modulates habituation rate: Evidence from a cross-modal auditory distraction paradigm. Psychonomic Bulletin \& Review, 19, 245-259.

Sörqvist, P., \& Rönnberg, J. (2012). Episodic long-term memory of spoken discourse masked by speech: What is the role for working memory capacity? Journal of Speech, Language, and Hearing Research, 55, 210-218.

Stenfelt, S., \& Rönnberg, J. (2009). The signal-cognition interface: Interactions between degraded auditory signals and cognitive processes. Scandinavian Journal of Psychology, 50, 385-393.

Treisman, A. (1964). Selective attention in man. British Medical Bulletin, 20, 12-16.

Turner, M. L., \& Engle, R. W. (1989). Is working memory capacity task dependent? Journal of Memory \& Language, 28, 127-154.

Wickens, C. D. (2008). Multiple resources and mental workload. Human Factors: The Journal of the Human Factors and Ergonomics, 50, 449-455.

Zhang, P., Chen, X., Yuan, P., Zhang, D., \& He, S. (2006). The effects of visuospatial attentional load on the processing of irrelevant acoustic distractors. Neuroimage, 33, 715-724. 